\title{
Evaluation of right adrenal vein cannulation by computed tomography angiography in 140 consecutive patients undergoing adrenal venous sampling
}

\section{Shiro Onozawa ${ }^{1}$, Satoru Murata ${ }^{1}$, Hiroyuki Tajima ${ }^{1}$, Hidenori Yamaguchi ${ }^{1}$, Takahiko Mine ${ }^{1}$, Akira Ishizaki ${ }^{2}$, Hitoshi Sugihara ${ }^{2}$, Shinichi Oikawa ${ }^{2}$ and Shin-ichiro Kumita'}

${ }^{1}$ Department of Radiology/Center for Advanced Medical Technology and 2Division of Diabetes, Endocrinology and Metabolism, Department of Medicine, Nippon Medical School, 1-1-5 Sendagi, Bunkyo-ku, Tokyo 113-8603, Japan
Correspondence

should be addressed

to S Onozawa

Email

onozawa@nms.ac.jp

\begin{abstract}
Objective: As it is now known that primary aldosteronism (PA) is more prevalent than was previously recognized, and is a potentially curable cause of hypertension and related cardiovascular diseases, the search for a safe and effective means of its diagnosis has reemerged as a topic of interest. Adrenal venous sampling is the gold standard for diagnosis of PA, but the technique is challenging and the small right adrenal vein can be particularly difficult to cannulate. Our objective was to evaluate the usefulness of computed tomography during angiography (angio-CT) in increasing the success of adrenal venous sampling and to identify factors associated with cannulation failure.

Design: Retrospective review.

Methods: A total of 140 consecutive patients with suspected PA except Cushing's syndrome treated at a single hospital from June 2008 to May 2013 were included. Catheter misplacement and correct cannulation rates before angio-CT and success rate of sampling after angio-CT were calculated. Univariate analysis for factors related to incorrect cannulation included gender, age, height, weight, BMI, and adrenal nodules. Successful sampling was biochemically defined according to cortisol concentrations in the venous blood samples.

Results: Angio-CT detected misplaced catheters in 13 patients (9.3\%). The calculated correct cannulation rate of adrenal vein sampling increased from $86.4 \%$ before angio-CT to $95.7 \%$ after CT $(P<0.001$, McNemar's test). Univariate analysis showed a tendency for a higher rate of failure of right adrenal venous sampling in taller patients ( $P=0.052$, Mann-Whitney's $U$ test). Conclusion: Angio-CT improved success of adrenal venous sampling.
\end{abstract}

\section{Introduction}

Primary aldosteronism (PA) is recognized as the most common cause of secondary hypertension. PA can result from an aldosterone-producing adenoma, bilateral idiopathic adrenal hyperplasia, unilateral adrenal hyperplasia, and other causes $(1,2)$. The prevalence of PA among patients with hypertension is now thought to be as high as $11 \%$. Patients with PA caused by unilateral hyperaldosteronism have an indication for surgical treatment, which can cure or alleviate hypertension and reduce the risk of cardiovascular complications (3). Adrenal venous sampling (AVS) is regarded as a gold standard for confirming the laterality of hyperaldosteronism in the diagnosis of PA, and a successful AVS can provide definitive guidance for further treatment options.

AVS was introduced by Melby (4) in the late 1960s as a test to distinguish unilateral from bilateral PA, but because (c) 2014 European Society of Endocrinology Printed in Great Britain
Published by Bioscientifica Ltd. 
of its difficulty, the procedure was not commonly used, and it was later thought that computed tomography (CT) and magnetic resonance imaging (MRI) would be adequate to accurately diagnose adrenal lesions. However, it is now reported that up to $37.8 \%$ of these lesions may be missed by traditional CT or MRI (5). Furthermore, CT and MRI cannot provide functional information about the adrenal glands. Thus, the potential contribution of AVS has once again been recognized, and the procedure seems to be regaining popularity as a definitive mode of lateralization of aldosteronism by direct measurement of adrenal aldosterone secretion.

Accurate sampling during AVS is mandatory for correct diagnosis and treatment of PA. The primary challenge in AVS is obtaining the samples from the right adrenal vein, because the right adrenal vein is small and has a complicated anatomy, and there is a high risk of displacement of the cannula due to respiratory motion. The success rates of right-AVS vary $(6,7,8)$, and have been reported to range from 55 to $98 \%$.

Recently, the usefulness of both C-arm $(9,10)$ and Dyna-CT (11) during AVS has been reported. CT can be used during AVS to provide visual confirmation of correct cannula placement and to reduce the risk of failed sampling, particularly from the right adrenal vein. CT during angiography (angio-CT) is a system that combines traditional angiography techniques with CT imaging. Results of angio-CT in tumor treatment are reported $(12,13)$, and in our institution, we have also been using angio-CT for AVS, in particular to confirm correct cannulation of the right adrenal vein.

The purpose of this was to assess the usefulness of angio-CT for facilitating correct cannulation of the right adrenal vein during AVS and to perform univariate analysis to identify factors that could contribute to failed right-AVS.

\section{Subjects and methods}

\section{Patients}

From June 2008 to May 2013, 148 consecutive patients who were diagnosed with PA according to the guideline of the Japan Endocrine Society (14), which recommends confirmation by at least two of three tests (captoprilchallenge test, upright furosemide-loading test, and saline-loading test), underwent AVS. Eight of the patients who were diagnosed with Cushing's syndrome or subclinical Cushing's syndrome were excluded from the data analysis. Before AVS, all antihypertensives were changed to calcium channel blockers or $\alpha$-blockers in accordance with the guideline of the Japan Endocrine Society (14). The study was approved by the local ethics committee and written informed consent was obtained from all patients whose medical records were reviewed for this study.

\section{Adrenal vein cannulation and cosyntropin stimulation}

Patients were prepared in the usual fashion, and 5-French (Fr) and 6-Fr introducer sheaths were inserted bilaterally into the femoral veins using the Seldinger technique. An initial sample of $3 \mathrm{ml}$ of venous blood was collected from the inferior vena cava (IVC) and then a 5-Fr catheter designed for left-AVS (Hanako, Tokyo, Japan) was inserted via the 5-Fr introducer in the left groin and advanced into the left adrenal vein. A high-flow microcatheter (Progreat Omega, Terumo, Tokyo, Japan) was also advanced into the left adrenal branch over a micro-guide wire (Aqua ${ }^{\circledR}$ VIII, Filmecc Co.Ltd, Nagoya, Japan), and was used to collect a 3-ml sample of left adrenal venous blood before cosyntropin stimulation, which will be further described below. A second 5-Fr catheter, designed especially for right-AVS (Hanako), was inserted via the 6-Fr introducer in the right groin. This catheter was carefully advanced and a contrast angiogram was obtained to check the catheter location in the right adrenal vein (Fig. 1A) in posteroanterior view. Then the retrograde right adrenal venography was routinely performed in right anterior oblique view $\left(30^{\circ}\right)$. If we determined that the first catheter was incorrectly placed, we attempted cannulation using other specialized catheters including Shepherd hook-type catheters or Cobra-type catheters (Medikit, Tokyo, Japan). Once the vessel believed to be the right adrenal vein was cannulated, a microcatheter with a tip incision designed for AVS (Goldcrest, Kosin Medical, Tokyo, Japan) and a microguidewire were inserted into a branch of the vessel (15) (Fig. 1B), and angio-CT, described in greater detail below, was performed in order to check for and confirm accurate placement of the right adrenal vein cannula. Repeat angiograms were obtained after angio-CT in every case to confirm that the catheter had not migrated during preparation or performance of the angio-CT examination. If a misplaced catheter was observed on angio-CT, repeat cannulation of the right adrenal vein was attempted and confirmed by the same procedures until it appeared that appropriate placement had occurred. After this, a 3-ml sample of blood was then taken from the right side before cosyntropin stimulation. 
Cosyntropin stimulation was carried out by injecting $0.25 \mathrm{mg}$ cosyntropin via a peripheral venous line. During cosyntropin stimulation, the microcatheters in both adrenal veins were filled with heparin sodium (500 U/each catheter) to avoid coagulation. Within 15-45 min after peripheral cosyntropin injection, repeat samples were taken from the adrenal veins and, using a 6-Fr introducer, from the IVC. Data from precosyntropin stimulation specimens were used as reference data, specifically in cases of AVS failure, and postcosyntropin stimulation results were used for data analysis in this study.
A

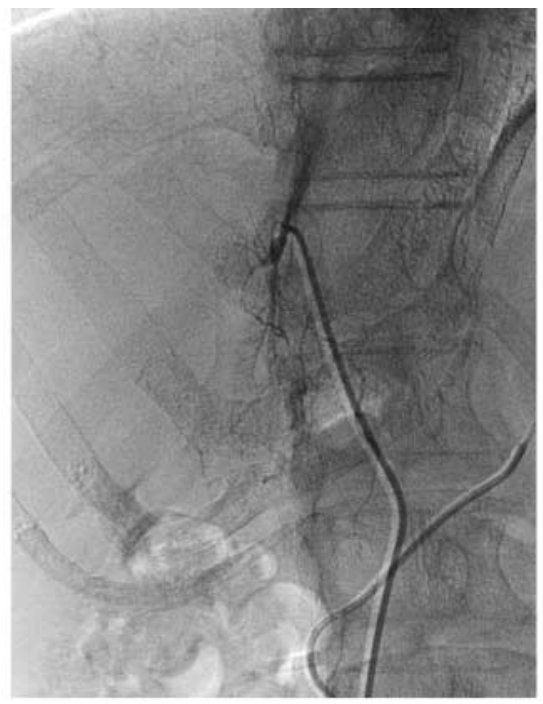

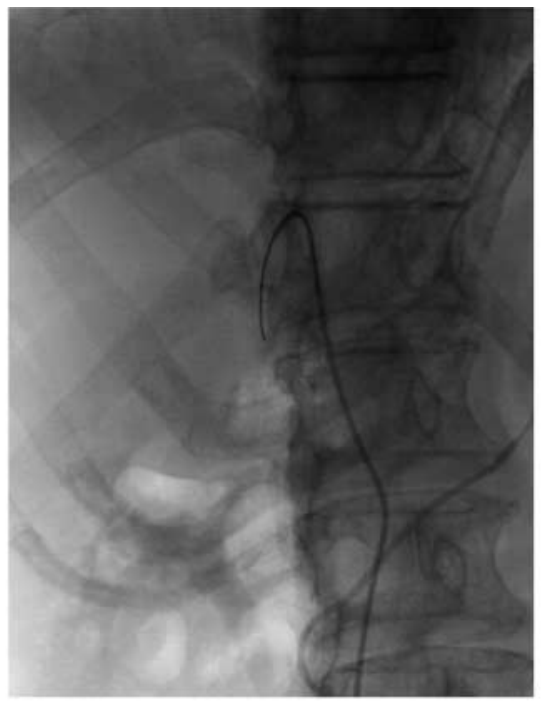

C

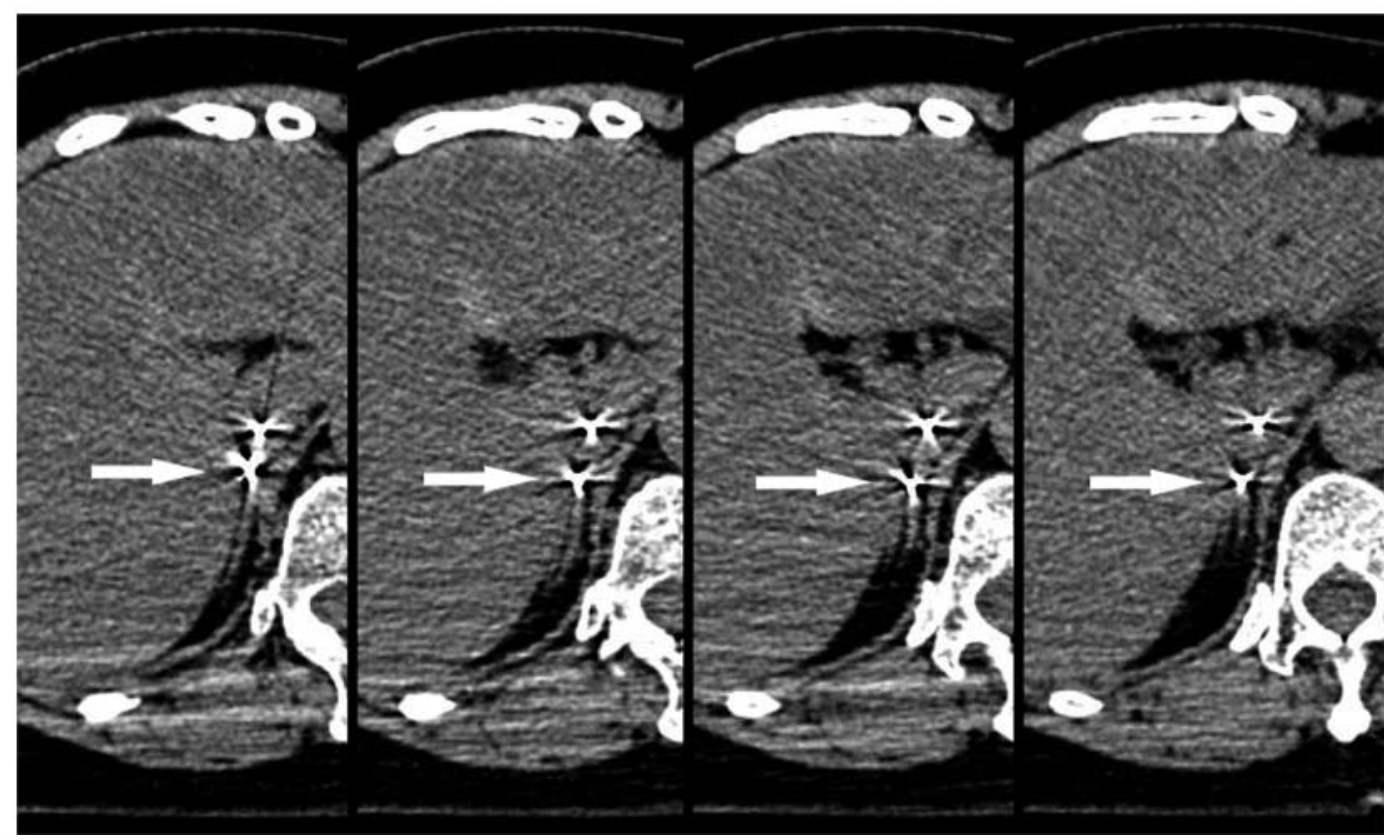

\section{Figure 1}

Angiography and angio-CT during adrenal venous sampling. (A) Angiography: retrograde right adrenal venography with contrast. (B) X-ray photogram: the micro-guidewire and microcatheter were inserted far into the right adrenal vein after angiography and before angio-CT to prevent displacement of the catheter. (C) Angio-CT during adrenal venous sampling: the high-density area on the right is the micro-guidewire (arrow) in the right adrenal vein. 


\section{Technical strategies for adrenal venous sampling}

Various specialized devices, including the special microcatheters described earlier, were available for use during the AVS procedures (15). We also used $10 \mathrm{ml}$ syringes containing $3 \mathrm{ml}$ of air to decrease aspiration pressures during sample collection (8). In order to reduce the potential for displacement of the adrenal vein cannula due to respiratory motion, we asked patients to avoid deep breathing and conversation during sampling and, when necessary, we provided supplemental oxygen. The small incisions at the tip of the special microcatheters designed for AVS are meant to prevent invagination of the venous walls.

\section{Angio-CT specifications and image evaluation}

A ROBUSTO 4-MDCT scanner was used for angio-CT (Hitachi Medical Corporation). Scans were obtained with the following parameters: 0.8 s per rotation; 1.25 collimations; $4.69 \mathrm{~mm} / \mathrm{s}$ table increment (pitch, 3.0); tube voltage, $120 \mathrm{kV}$; and tube current, $350 \mathrm{~mA}$. The patients were positioned so that their spine was near the iso-center of the CT gantry. The scans were performed without breath holding. Plain X-ray CT scans were obtained with the micro-guidewire in the right adrenal vein (Fig. 1C) to stabilize the microcatheter and to avoid contrast injection. It is known that a strong injection force can cause adrenal venous rupture (8). Transverse sections were reconstructed with a $1.25 \mathrm{~mm}$ section thickness at $1.25 \mathrm{~mm}$ intervals. The reconstruction field of view was set to $25 \mathrm{~cm}$ around the aorta.

Images were interpreted with a diagnostic-based viewer (We-view, Hitachi Medical Corporation) and only a cine-mode display of the transverse images was used to evaluate the presumed right adrenal vein. Two boardcertified radiology specialists (12 and 8 years of experience respectively) analyzed the imaging studies and in cases of disagreement, a final consensus was reached though inter-observer discussion.

\section{Confirmation of successful AVS: selectivity index}

Successful AVS via correct cannulation of adrenal veins was ultimately defined by adrenal venous cortisol concentration after cosyntropin stimulation, which should be $>200 \mu \mathrm{g} / \mathrm{dl}$ or greater than or equal to fivefold the cortisol concentration in the IVC specimen, as per the guideline of the Japan Endocrine Society (14).
Laterality of hyperaldosteronism: lateralization index

Laterality of hyperaldosteronism was confirmed if the aldosterone:cortisol concentration ratio on the side with the greater value was $\geq 2$.6-fold than that of the side with the lesser value. These definitions were also based on the guidelines of the Japan Endocrine Society (14).

\section{Calculation of correct cannulation rates before angio-CT and success rates after angio-CT of AVS}

We calculated the ratio of misplaced catheters detected by angio-CT and the correct cannulation rate of right AVS before angio-CT. The correct cannulation rate before angio-CT was calculated as: (number of radiolographically successful procedures of AVS) - (number of radiographically misplaced catheters detected by angio-CT)/(140 (i.e. the total number of AVS cases)). We also calculated the success rate of both sides of AVS after angio-CT confirmation.

We compared the correct cannulation of the adrenal vein before angio-CT and success rate of AVS after angioCT on the right side and both sides.

\section{Univariate analysis}

We also sought factors related to correct and incorrect cannulation before angio-CT via univariate analysis of gender, age, height, weight, BMI, and presence of adrenal nodules $\geq 5 \mathrm{~mm}$ in diameter confirmed by CT within 3 months of AVS.

\section{Statistical analysis}

$\chi^{2}$-test was used to compare the difference between categorical variables, and Mann-Whitney's $U$ test was used to compare the difference between nonparametrically distributed continuous variables. $P$ values of $<0.05$ were considered to be statistically significant. PASW Statistics 18 software (SPSS, Inc.) was used for all statistical analyses.

\section{Results}

A total of 140 patients, 51 males and 89 females, median age 56.0 years (interquartile range (IQR): 18 years), were included in the analysis. Patient characteristics are presented in detail in Table 1.

Angio-CT detected misplaced catheters in 13/140 patients (9.3\%). AVS was biochemically successful after 
Table 1 Patients characteristics.

\begin{tabular}{|c|c|}
\hline \multicolumn{2}{|l|}{ Gender } \\
\hline Male & 51 \\
\hline Female & 89 \\
\hline Age (median (IQR)) & $56.0(18.0)$ \\
\hline Height $(\mathrm{cm})$ & $160.0(12.5)$ \\
\hline Weight (kg) & $64.0(16.1)$ \\
\hline BMI & $25.0(5.5)$ \\
\hline \multicolumn{2}{|l|}{ Nodules } \\
\hline None & 87 \\
\hline Right side & 18 \\
\hline Left side & 28 \\
\hline Both sides & 7 \\
\hline Systolic BP (mmHg) & $142.0(29.0)$ \\
\hline Diastolic BP (mmHg) & $82.0(22.0)$ \\
\hline Aldosterone $(\mathrm{pg} / \mathrm{ml})$ & $148.3(107.0)$ \\
\hline Plasma renin activity (ng/ml per hour) & $0.3(0.3)$ \\
\hline Aldosterone:renin ratio $\left(10^{-3} \mathrm{~h}\right)$ & $391.0(695.0)$ \\
\hline Potassium (mEq/l) & $3.8(0.6)$ \\
\hline
\end{tabular}

$\mathrm{IQR}$, interquartile range; $\mathrm{BP}$, blood pressure.

angio-CT in 134 of 140 patients (95.7\%) overall, with biochemically successful right-AVS in 135 of 140 (96.4\%) patients and biochemically successful left-AVS in 139 of $140(99.3 \%)$ patients.

Sites of misplaced catheters were the inferior accessory hepatic veins in five cases and the renal capsular veins in five cases, and there were three cases of catheter migration into the IVC. All misplaced right AVS catheters detected by angio-CT were correctly repositioned and confirmed by repeat angio-CT. The calculated correct cannulation rate of bilateral adrenal veins before angio-CT was 86.4\% (121 of 140 patients, $P<0.001$ ) and the calculated correct cannulation rate of right adrenal vein before angio-CT was $87.1 \%$ (122 of 140 patients, $P<0.001$ ).

When we performed the univariate analysis of factors associated with correct or incorrect cannulation of right adrenal vein before angio-CT, there were no significant differences among the parameters evaluated (Table 2), although there was a trend $(P=0.052)$ toward higher incorrect cannulation rate in taller patients. As a final result of AVS, unilateral hyperaldosteronism was confirmed in 30 of 140 cases. Twenty patients had excess aldosterone secretion from the right adrenal gland (14.9\%) and 10 (7.5\%) from the left adrenal gland; surgery was recommended for all 30 of these patients. These details are summarized in Fig. 2.

\section{Discussion}

The selectivity index is generally regarded as the main determinant of a successful AVS procedure (5), with cutoff values ranging $>1.1$ to $>5$. A cutoff value of $>5$ was used in this study (adrenal vein cortisol concentration of $>200 \mu \mathrm{g} / \mathrm{dl}$ ), which was established in accordance with the guidelines of the Japan Endocrine Society (14). As noted, success rates of right AVS ranging from 55 to $98 \%$ have been reported $(6,7,8)$. Although the wide range of accepted cutoff values for the selectivity index makes it difficult to directly compare the results of these studies, we believe that our overall success rate of angio-CT-assisted AVS of $95.7 \%$ affirms the value of angio-CT for reducing technical failures during cannulation of the right adrenal vein. The median value of the right-selectivity index among the five patients in whom right AVS procedures failed was 1.5 (IQR 1.2); therefore, even with the selectivity index reduced to a value of $>3$, all of these cases would have been classified as failures. On the other hand, the selectivity index in the patient in whom left AVS failed was 4.18, and the procedure would have been categorized as a success if we had chosen the lower cutoff value, which further highlights the difficulty of comparing AVS success rates among reports.

There are also different criteria for the diagnosis of PA including its subtype, different criteria for lateralization index, and usage of cosyntropin stimulation. Especially, the use of cosyntropin stimulation for AVS also remains a subject of debate. Monticone et al. (16) have asserted that cosyntropin stimulation is useless for AVS in centers with high success rates; once again, our choice to use cosyntropin stimulation in the current study was due to

Table 2 Comparison between success and failure before angio-CT.

\begin{tabular}{|c|c|c|c|}
\hline & $\begin{array}{l}\text { Success } \\
(n=122)\end{array}$ & $\begin{array}{l}\text { Failure } \\
(n=18)\end{array}$ & $\boldsymbol{P}$ \\
\hline \multicolumn{4}{|l|}{ Gender } \\
\hline Male & 44 & 7 & $0.816^{*}$ \\
\hline Female & 78 & 11 & \\
\hline Age (median (IQR)) & $56.0(18)$ & $55.0(14)$ & 0.963 \\
\hline Height & $159.0(12.2)$ & $166.5(12.4)$ & 0.052 \\
\hline Weight & $63.8(16.2)$ & $67.5(28.6)$ & 0.278 \\
\hline BMI & $25.0(5.31)$ & $24.3(8.0)$ & 1.000 \\
\hline \multicolumn{4}{|l|}{ Nodules } \\
\hline None & 77 & 10 & $0.641 *$ \\
\hline Right side & 14 & 4 & \\
\hline Left side & 25 & 3 & \\
\hline Both sides & 6 & 1 & \\
\hline $\begin{array}{l}\text { Aldosterone:cortisol ratio } \\
\text { before cosyntropin in IVC }\end{array}$ & $8.9(7.5)$ & NA & \\
\hline $\begin{array}{l}\text { Aldosterone:cortisol ratio } \\
\text { after cosyntropin in IVC }\end{array}$ & $9.3(6.3)$ & NA & - \\
\hline Selectivity index in right side & $47.4(26.9)$ & NA & - \\
\hline Selectivity index in left side & $36.4(20.1)$ & NA & - \\
\hline
\end{tabular}

IQR, interquartile range; NA, not available; $P$ : $P$ value (Mann-Whitney's $U$ test and $\chi^{2}$-test*). 


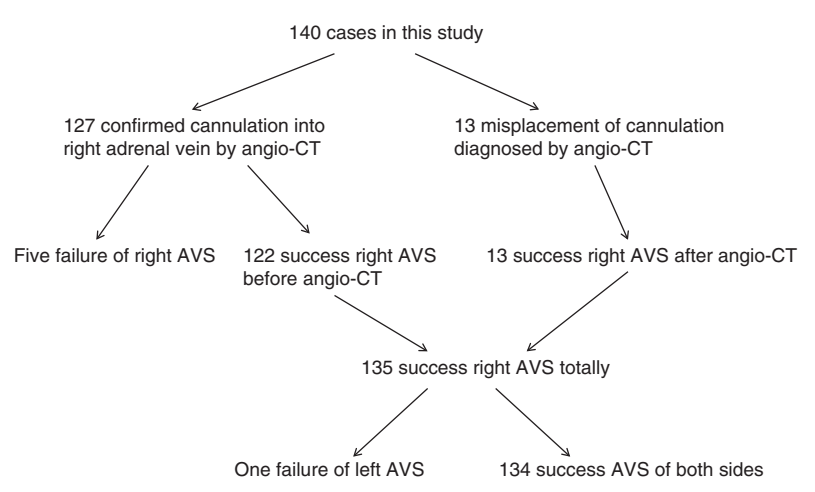

Figure 2

Details of right adrenal venous sampling. The calculated success rate before angio-CT was $87.1 \%$. Misplaced catheters were detected by angio-CT in 13/140 patients (9.3\%). AVS ultimately failed in five patients (3.6\%). Catheters had appeared to be correctly placed on angio-CT in all five.

guidelines of the Japan Endocrine Society (14). We plan to investigate the possibility of AVS without cosyntropin stimulation using pre-cosyntropin stimulation data collected during this study.

Other CT techniques that have been used during AVS include C-arm CT $(9,10)$ and Dyna-CT $(11)$, both of which have also proven to reduce technical failure of right adrenal vein cannulation. The overall rate of misplacement of catheters intended for the right adrenal vein was $\sim 20 \%$ in these reports. Angio-CT is a combination of angio-suites and CT. Compared with Dyna-CT or C-arm $\mathrm{CT}$, angio-CT provides superior low-contrast resolution, wider field of view, and better signal-to-noise ratios. In this study, the rate of correct cannulation of the right adrenal vein was $87.1 \%$ before angio-CT and $96.4 \%$ after angio-CT. Thus, angio-CT allowed immediate observation of catheter placement with markedly improved success rates.

Although angio-CT is useful to confirm cannulation of the right adrenal vein and to reduce technical failures during AVS, it is important that patient movement, conversation, deep breathing, and breath holding, which may all cause displacement of the catheter, as well venous injury due to high-pressure contrast medium injection, are avoided. In our technique, we also seek to reduce the risk of catheter migration due to patient movement by asking patients to keep their arms at their sides. In addition, to further stabilize the microcatheter in the right adrenal vein, we advanced the micro-guidewire a good distance into the branch of the adrenal vein, which also allowed us to eliminate the risk of venous injury due to high-pressure contrast injection, because we were able to use the guide wire, rather than contrast medium, to confirm the location of the vessel. We obtained angiograms of the cannulated adrenal veins before and after aspiration of blood samples to further reduce the likelihood of failed AVS procedures.

Regarding the five cases of failed right AVS in the present series, we speculated that two of them were due to collection of specimens from the confluence of the smaller right adrenal branches into the wider renal capsular veins, rather than from the adrenal branches. In another case, we suspected that the patient had no main adrenal vein, and a second sampling performed via small collateral veins was successful. The last two failed cases were thought to be due to right adrenal veins that were too narrow or too small to allow stabilization of the catheters, which probably resulted in catheter migration during the sampling procedure. To avoid unnecessary repeat AVS procedures in these cases, we performed blood sampling from several locations, and included selective venous sampling when possible.

Although we could not identify any specific factors that may affect the success of AVS by our univariate analysis, based on our finding that the major route of misplacement of catheters in the present series involved the inferior accessory hepatic veins or the capsular renal veins, and based on other reports about the relationship between the right adrenal vein and the inferior accessory hepatic vein, which may help or hinder the AVS due to its location or size $(17,18,19)$, we speculated that the trend toward a higher rate of AVS failure in taller patients may have been because taller patients could have smaller inferior accessory hepatic veins or wider renal capsular veins than shorter patients. However, the available data does not allow us to validate this tendency.

Finally, we believe that the special microcatheters with tip incision that were used in this study (15) to avoid invagination in the walls of the small branches of the adrenal vein during selective sampling contributed to our success rate. Omura et al. (20) have also reported the advantage of selective venous sampling in a series of patients with multiple unilateral adrenocortical micronodules. Use of these microcatheters for AVS is helpful for aspirating blood from the small adrenal veins, but we could not overlook the possibility of ultra-selective sampling from a particular branch of the adrenal vein. Therefore, it was important to confirm catheter placement in the main adrenal vein.

There were some limitations in this study. First, this study was not a randomized controlled study and all 
procedures were performed in a single center. To more adequately assess the value of angio-CT in AVS, a prospective randomized multicenter comparison of AVS with and without angio-CT would be necessary. Second, we did not examine the specific technical difficulties of each case. There were difficult cases in this series related to anatomy or breath motion. A multivariate analysis with a much larger group of patients would be required to identify specific technical factors that can be related to success or failure of AVS. Third, we did not assess contrastenhanced CT before AVS in this study. While some patients did have enhanced CT, which might have improved the results of AVS, we determined that inclusion of the combination of enhanced CT and angio-CT was inappropriate for this study protocol. Finally, each angioCT exam exposes the patient to $2.7 \mathrm{mSv}$ of additional radiation. Therefore, although it is useful, angio-CT should be reserved for cases in which cannulation cannot be confirmed during routine angiography.

In conclusion, we found that angio-CT guidance increases the success rate of AVS with angio-CT, and is especially useful for facilitating correct cannulation of the right adrenal vein. However, angio-CT must be used judiciously in order to avoid unnecessary radiation exposure.

\section{Declaration of interest}

The authors declare that there is no conflict of interest that could be perceived as prejudicing the impartiality of the research reported.

Funding

This work was supported by JSPS KAKENHI grant number 25861130 .

\section{Author contribution statement}

S Onozawa, S Murata, and S Kumita conceived and designed the study. S Onozawa and $\mathrm{H}$ Tajima wrote the draft of the manuscript. S Onozawa, $\mathrm{H}$ Yamaguchi, and $\mathrm{T}$ Mine performed the interventions and collected the data. S Onozawa and A Ishizaki analyzed and interpreted the data. S Onozawa and S Murata made critical revision of the article for important intellectual content. S Kumita and S Oikawa made final approval of the article. All of the authors discussed the results and commented on the manuscript.

\section{References}

1 Rossi GP, Bernini G, Caliumi C, Desideri G, Fabris B, Ferri C, Ganzaroli C, Giacchetti G, Letizia C, Maccario M et al. A prospective study of the prevalence of primary aldosteronism in 1,125 hypertensive patients. Journal of the American College of Cardiology 200648 2293-2300. (doi:10.1016/j.jacc.2006.07.059)
2 Douma S, Petidis K, Doumas M, Papaefthimiou P, Triantafyllou A, Kartali N, Papadopoulos N, Vogiatzis K \& Zamboulis C. Prevalence of primary hyperaldosteronism in resistant hypertension: a retrospective observational study. Lancet 2008371 1921-1926. (doi:10.1016/S01406736(08)60834-X)

3 Obara T, Ito Y, Okamoto T, Kanaji Y, Yamashita T, Aiba M \& Fujimoto Y. Risk factors associated with postoperative persistent hypertension in patients with primary aldosteronism. Surgery 1992112 987-993.

4 Melby JC. Diagnosis and localization of aldosterone-producing adenomas by adrenal-vein catheterization. New England Journal of Medicine 1967277 1050-1056. (doi:10.1056/NEJM196711162772002)

5 Kempers MJ, Lenders JW, van Outheusden L, van der Wilt GJ, Schultze Kool LJ, Hermus AR \& Deinum J. Systematic review: diagnostic procedures to differentiate unilateral from bilateral adrenal abnormality in primary aldosteronism. Annals of Internal Medicine $2009 \mathbf{1 5 1}$ 329-337. (doi:10.7326/0003-4819-151-5-200909010-00007)

6 Auchus RJ, Michaelis C, Wians FH Jr, Dolmatch BL, Josephs SC, Trimmer CK, Anderson ME \& Nwariaku FE. Rapid cortisol assays improve the success rate of adrenal vein sampling for primary aldosteronism. Annals of Surgery 2009249 318-321. (doi:10.1097/SLA. Ob013e3181961d77)

7 Young WF Jr \& Klee GG. Primary aldosteronism. Diagnostic evaluation. Endocrinology and Metabolism Clinics of North America 198817 367-395.

8 Daunt N. Adrenal vein sampling: how to make it quick, easy, and successful. Radiographics 200525 (Suppl 1) S143-S158. (doi:10.1148/rg. 25si055514)

9 Georgiades CS, Hong K, Geschwind JF, Liddell R, Syed L, Kharlip J \& Arepally A. Adjunctive use of C-arm CT may eliminate technical failure in adrenal vein sampling. Journal of Vascular and Interventional Radiology 200718 1102-1105. (doi:10.1016/j.jvir.2007.06.018)

10 Kinnison M. Adrenal vein sampling with C-arm CT. Journal of Vascular and Interventional Radiology 200819153 author reply 153. (doi:10.1016/ j.jvir.2007.10.004)

11 Plank C, Wolf F, Langenberger H, Loewe C, Schoder M \& Lammer J. Adrenal venous sampling using Dyna-CT - a practical guide. European Journal of Radiology 201281 2304-2307. (doi:10.1016/j.ejrad.2011. 05.011)

12 Ishikura R, Ando K, Nagami Y, Yamamoto S, Miura K, Pande AR, Yamano T, Hirota S \& Nakao N. Evaluation of vascular supply with cone-beam computed tomography during intraarterial chemotherapy for a skull base tumor. Radiation Medicine 200624 384-387. (doi:10.1007/s11604-006-0038-x)

13 Yoshida K, Kobayashi S, Matsui O, Gabata T, Sanada J, Koda W, Minami T, Ryu Y, Kozaka K \& Kitao A. Hepatic pseudolymphoma: imaging-pathologic correlation with special reference to hemodynamic analysis. Abdominal Imaging 201338 1277-1285. (doi:10.1007/s00261-013-0016-6)

14 Nishikawa T, Omura M, Satoh F, Shibata H, Takahashi K, Tamura N, Tanabe A \& Task Force Committee on Primary Aldosteronism. The Japan Endocrine Society guidelines for the diagnosis and treatment of primary aldosteronism - the Japan Endocrine Society 2009. Endocrine Journal 201158 711-721. (doi:10.1507/endocrj. EJ11-0133)

15 Nishikawa T, Matsuzawa Y, Saito J \& Omura M. Is it possible to extirpate cardiovascular events in primary aldosteronism after surgical treatment? Japanese Clinical Medicine 20101 21-23. (doi:10.4137/ JCM.S6316)

16 Monticone S, Satoh F, Giacchetti G, Viola A, Morimoto R, Kudo M, Iwakura Y, Ono Y, Turchi F, Paci E et al. Effect of adrenocorticotropic hormone stimulation during adrenal vein sampling in primary aldosteronism. Hypertension 201259 840-846. (doi:10.1161/ HYPERTENSIONAHA.111.189548)

17 Matsuura T, Takase K, Ota H, Yamada T, Sato A, Satoh F \& Takahashi S. Radiologic anatomy of the right adrenal vein: preliminary experience with MDCT. American Journal of Roentgenology 2008191 402-408. (doi:10.2214/AJR.07.3338) 
18 Trerotola SO, Smoger DL, Cohen DL \& Fraker DL. The inferior accessory hepatic vein: an anatomic landmark in adrenal vein sampling. Journal of Vascular and Interventional Radiology 201122 1306-1311. (doi:10.1016/j.jvir.2010.12.040)

19 Miotto D, De Toni R, Pitter G, Seccia TM, Motta R, Vincenzi M, Feltrin G $\&$ Rossi GP. Impact of accessory hepatic veins on adrenal vein sampling for identification of surgically curable primary aldosteronism.
Hypertension 200954 885-889. (doi:10.1161/HYPERTENSIONAHA.109. 134759)

20 Omura M, Sasano H, Fujiwara T, Yamaguchi K \& Nishikawa T. Unique cases of unilateral hyperaldosteronemia due to multiple adrenocortical micronodules, which can only be detected by selective adrenal venous sampling. Metabolism 200251 350-355. (doi:10.1053/ meta.2002.30498)

Received 10 September 2013

Revised version received 21 January 2014

Accepted 23 January 2014 